\title{
Impact of coronavirus disease 2019 on pulmonary function in early convalescence phase
}

Yiying Huang ${ }^{1,2+}$, Cuiyan Tan ${ }^{1 \dagger}$, Jian $\mathrm{Wu}^{1 \dagger}$, Meizhu Chen ${ }^{1}$, Zhenguo Wang ${ }^{1}$, Liyun Luo ${ }^{3}$, Xiaorong Zhou', Xinran Liu', Xiaoling Huang ${ }^{1}$, Shican Yuan ${ }^{1}$, Chaolin Chen ${ }^{1}$, Fen Gao ${ }^{1}$, Jin Huang ${ }^{1}$, Hong Shan ${ }^{2}$ and Jing Liu Li $^{1}$ (D) $^{*}$

\begin{abstract}
Objective: This study investigated the influence of Coronavirus Disease 2019 (COVID-19) on lung function in early convalescence phase.

Methods: A retrospective study of COVID-19 patients at the Fifth Affiliated Hospital of Sun Yat-sen University were conducted, with serial assessments including lung volumes (TLC), spirometry (FVC, FEV1), lung diffusing capacity for carbon monoxide (DLCO),respiratory muscle strength, 6-min walking distance (6MWD) and high resolution CT being collected at 30 days after discharged.

Results: Fifty-seven patients completed the serial assessments. There were 40 non-severe cases and 17 severe cases. Thirty-one patients (54.3\%) had abnormal CT findings. Abnormalities were detected in the pulmonary function tests in 43 (75.4\%) of the patients. Six (10.5\%), 5(8.7\%), 25(43.8\%) 7(12.3\%), and 30 (52.6\%) patients had FVC, FEV1, FEV1/FVC ratio, TLC, and DLCO values less than $80 \%$ of predicted values, respectively. 28 (49.1\%) and $13(22.8 \%)$ patients had PImax and PEmax values less than $80 \%$ of the corresponding predicted values. Compared with non-severe cases, severe patients showed higher incidence of DLCO impairment (75.6\%vs $42.5 \%, p=0.019)$, higher lung total severity score (TSS) and R20, and significantly lower percentage of predicted TLC and 6MWD. No significant correlation between TSS and pulmonary function parameters was found during follow-up visit.

Conclusion: Impaired diffusing-capacity, lower respiratory muscle strength, and lung imaging abnormalities were detected in more than half of the COVID-19 patients in early convalescence phase. Compared with non-severe cases, severe patients had a higher incidence of DLCO impairment and encountered more TLC decrease and 6MWD decline.
\end{abstract}

Keywords: COVID-19, Early convalescence, Lung function, Respiratory muscle strength

\footnotetext{
* Correspondence: liujing25@mail.sysu.edu.cn

${ }^{\dagger}$ Yiying Huang, Cuiyan Tan and Jian Wu contributed equally to this work. 'Department of Pulmonary and Critical Care Medicine, The Fifth Affiliated Hospital of Sun Yat-sen University, 52 East Meihua Rd, Zhuhai City 519000, China

${ }^{2}$ Guangdong Provincial Key Laboratory of Biomedical Imaging, The Fifth Affiliated Hospital of Sun Yat-sen University, 52 East Meihua Rd, Zhuhai City 519000, China

Full list of author information is available at the end of the article
}

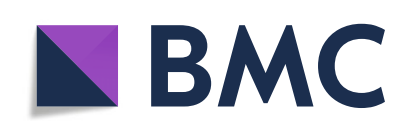

(c) The Author(s). 2020 Open Access This article is licensed under a Creative Commons Attribution 4.0 International License, which permits use, sharing, adaptation, distribution and reproduction in any medium or format, as long as you give appropriate credit to the original author(s) and the source, provide a link to the Creative Commons licence, and indicate if changes were made. The images or other third party material in this article are included in the article's Creative Commons licence, unless indicated otherwise in a credit line to the material. If material is not included in the article's Creative Commons licence and your intended use is not permitted by statutory regulation or exceeds the permitted use, you will need to obtain permission directly from the copyright holder. To view a copy of this licence, visit http://creativecommons.org/licenses/by/4.0/ The Creative Commons Public Domain Dedication waiver (http://creativecommons.org/publicdomain/zero/1.0/) applies to the data made available in this article, unless otherwise stated in a credit line to the data. 


\section{Background}

Coronavirus Disease 2019 (COVID-19) is a new and highly contagious respiratory disease caused by severe acute respiratory syndrome coronavirus 2 (SARS-CoV2 ), which presented a risk of infection from human to human [1]. The current outbreak of COVID-19 has caused a global pandemic. As of 7 June, 2020, there were $6,663,304$ confirmed cases and 392,802 confirmed deaths globally. It might progress rapidly, and some patients developed respiratory failure early in the disease. The knowledge about COVID-19, including clinical manifestations, pathogenesis, even treatment came from research and observation during the acute infection period $[2,3]$. In China, the vast majority of the patients had been successfully discharged. Until now, no study have reported early prognosis in relation to the degree of lung injury and rehabilitation in patients with COVID-19. Retrospective study showed that many patients had imaging abnormalities when discharged, a few patients even had pulmonary fibrosis. Lung function damage of patients with COVID-19 in early convalescence phase deserves attention. In order to have a more comprehensive understanding of the possible clinical outcomes of COVID-19, we conducted a retrospective study involving 57 discharged but undergoing rehabilitation COVID19 patients. Serial lung function, lung imaging examination and exercise capacity were examined at 30 days after discharged. In addition, we compared severe patients with non-severe patients by outcome parameters.

\section{Materials and methods Patient selection}

This is a follow up study of COVID-19 patients at 30 days after discharged from our hospital. From January 17, 2020 to March 1, 2020, a total of 103 COVID-19 patients were admitted to the Fifth Affiliated Hospital of Sun Yat-sen University. The diagnosis of COVID-19 was based on the CDC criteria. All patients had laboratory-confirmed SARS-CoV-2 infection by real-time reverse transcription polymerase chain reaction (RT-PCR) or next-generation sequencing. They all reached uniform discharge standard issued by the National Health Commission of China and had been released from the hospital over 1 month. In 30 days after discharged, patients were eligible to participate in the study if they were over 18 years of age. Patients with a previous history of pulmonary resection, neurological disease, or mental illness were excluded from our study. We obtained written informed consent from the patients before pulmonary function testing. This study was approved by the institutional ethics committee of the Fifth Affiliated Hospital of Sun Yat-sen University.

\section{Classification}

We retrospectively analyzed the medical records of these patients, and divided them into non-severe and severe groups according to the severity of the disease. Patients would be defined as severe cases if satisfied any of the following criteria: shortness of breath, $R R \geq 30$ times per minute; blood oxygen saturation $\leq 93 \%$ in resting state; partial arterial oxygen pressure $(\mathrm{PaO} 2) /$ fraction of inspiration $\mathrm{O} 2(\mathrm{Fi02}) \leq 300 \mathrm{mmmHg}$;respiratory failure requires mechanical ventilation; shock occurred or combined with other organ failure required ICU monitoring and treatment. Otherwise were mild cases.

\section{Lung imaging acquisition and CT quantitative evaluation}

All subjects underwent high resolution spiral CT (SOMATOM Definition Flash Siemens; Erlangen, Germany) scans in the supine position during endinspiration. Images were reconstructed at $1.0 \mathrm{~mm}$ slice thickness, with $1 \mathrm{~mm}$ increment, $512 \mathrm{~mm} \times 512 \mathrm{~mm}$. The images were assessed by two radiologists, both of whom were blinded to the clinical information. We used the same method as Michael et al., to quantify pulmonary inflammation severity [4-6]. Briefly, each of the five lung lobes was assessed for degree of involvement and classified as none (0\%), minimal (1-25\%), mild (26-50\%), moderate $(51-75 \%)$, or severe (76-100\%). No involvement corresponded to a lobe score of 0 , minimal involvement to a lobe score of 1 , mild involvement to a lobe score of 2 , moderate involvement to a lobe score of 3 , and severe involvement to a lobe score of 4.An overall lung "total severity score" was reached by summing the five lobe scores (range of possible scores, 0-20). All CT scores were independently performed by two respiratory doctors. Agreement was reached by consensus.

\section{6 min walk test}

Six min walk test (6MWT) is an exercise test that evaluates the functional status which is relevant to daily activities of patients with cardiopulmonary disease. The walking distance is closely related to gender, age and height, conventionally need a hierarchical analysis according to the above parameters. However, the sample size of our study was small which was not suitable for stratified analysis based on age, gender and height. So we estimated the walking distance of healthy people of the same gender, age and height according to reference equations for the $6 \mathrm{MWT}$ in healthy adults [7]. Then we calculated the ratio of measured value of the patients to the predicted value of the healthy person in fair condition. By comparing the ratio of two groups we could see whether there was difference in 6MWD between nonsevere and severe COVID-19 patients.

\section{Pulmonary function test and respiratory muscle strength measurement}

Each subject underwent a standard pulmonary function test (Master Screen, Jaeger, German). Recorded 
parameters include: total lung volume (TLC), forced vital capacity (FVC), residual volume (RV), forced expiratory volume in the first second (FEV1), maximum expiratory flow rate (MMEF75/25), FEV1 / FVC ratio, and diffusing capacity of the lung for carbon monoxide (DLCO). Impuse oscillation system (IOS) was used to measure airway viscosity resistance at an oscillation frequency of $5 \mathrm{~Hz}(\mathrm{R} 5)$, and central airway resistance at an oscillation frequency of $20 \mathrm{~Hz}$ (R20). Mouth pressure gauges can measure the maximum static inspiratory pressure (PImax) or maximum static expiratory pressure (PEmax) through a flanged cigarette holder. All subjects used this simple method to gauge inspiratory and expiratory muscle strength. The spirometry, DLCO, and respiratory muscle strength measurements were expressed as a percentage of predicted normal values.

To protect lung function laboratory staff, lung function tests were performed in a room with negative pressure device. Staff wore personal protective equipment, including N95 respirators, protective glasses, gloves and gowns. In addition, each patient used disposable virus and bacterial filters during the test.

\section{Statistics}

Statistical analysis was performed using Statistical Package for Social Science (SPSS) Version 13.0. Measurement data was expressed as mean \pm standard deviation. Continuous variables were compared using independent- sample t test, whereas the rank sum test was used for nonparametric data. Comparison of proportion was evaluated by Chi-square test. Spearman correlation test was used to detect the correlations between lung function and lung total severity score. All statistical tests were two tailed. Statistical significance was taken as $\mathrm{p}<0.05$.

\section{Results}

Characteristics of the enrolled COVID-19 patients

This study evaluated a total of 102 patients. Five patients were excluded for underage. Twenty-four patients were not included as it was less than 30 days after discharge. Three patients were excluded due to neurological or mental illness. In addition, eight patients had been out of contact. At last, 57 patients had been included and completed the serial assessments in the study (Fig. 1). There were 26 men and 31 women with a mean age of $46.72 \pm 13.78$ years (age range, 19 to 71 years), the mean body mass index was $23.99 \pm 3.55 \mathrm{~kg} / \mathrm{m} 2$.Among the 57 subjects, $46(80.7 \%)$ had a history of direct contact with Wuhan, Hubei. Nine patients(15.7\%)had a history of smoking. Twenty-one patients $(36.8 \%)$ had preexisting medical illness. The four most common preexisting illnesses were hypertension (11 patients), diabetes (four patients), malignant tumor (three patients) and cardiovascular disease (three patients). All of these conditions were either healed, or stable and well controlled at the

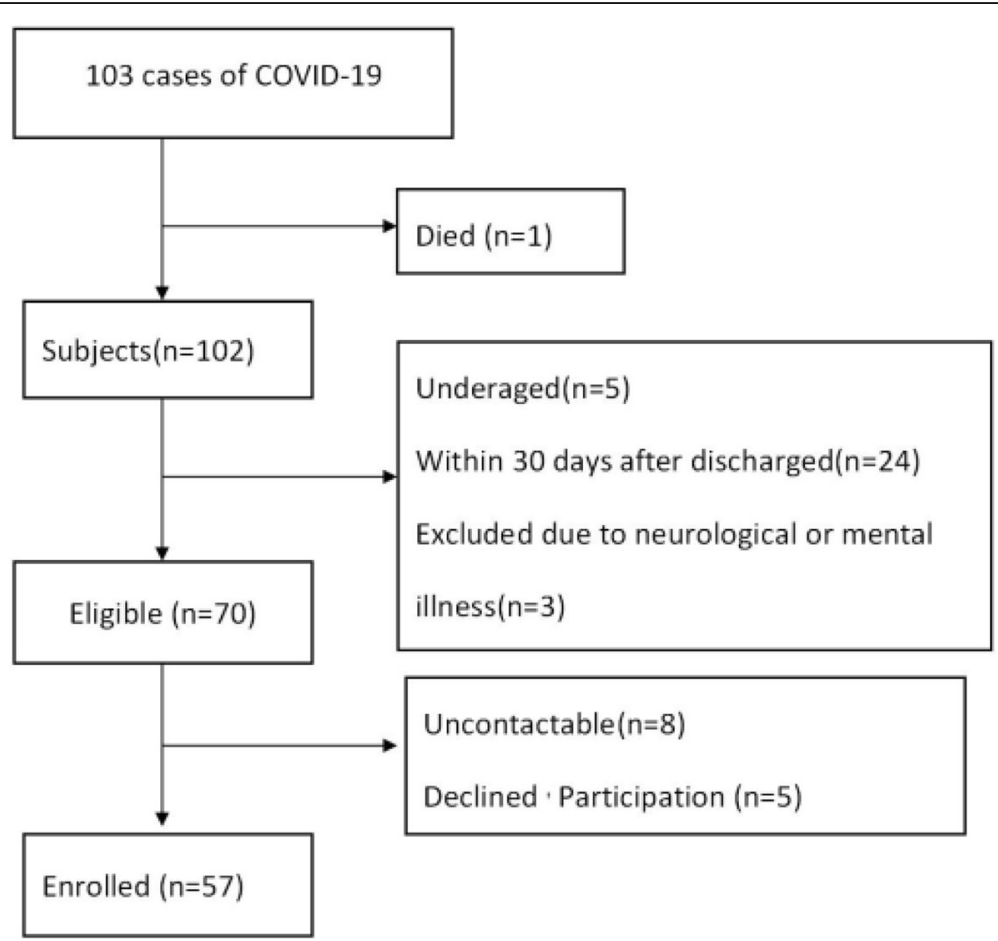

Fig. 1 Enrollment of COVID-19 patients in Early Convalescence 
Table 1 Demographic and clinical characteristic of COVID-19 patients

\begin{tabular}{|c|c|c|c|c|}
\hline Characteristic & Total $(n=57)$ & Severe $(n=17)$ & Non-severe $(n=40)$ & $p$ Value \\
\hline Age, year & $46.72(13.78)$ & $52.53(13.30)$ & $44.25(13.3)$ & 0.031 \\
\hline Male gender, No & $26(45.6 \%)$ & $12(70.6 \%)$ & $14(35.0 \%)$ & 0.014 \\
\hline $\mathrm{BMl}, \mathrm{kg} / \mathrm{m} 2$ & $23.99(3.55)$ & $25.54(3.43)$ & $23.33(3.42)$ & 0.103 \\
\hline Pre-existing medical illness & $21(36.8 \%)$ & $7(41.2 \%)$ & $14(35 \%)$ & 0.658 \\
\hline LOS, days & $20.89(7.22)$ & $20(16-24)$ & $19(15-24)$ & 0.834 \\
\hline $\mathrm{WBC}, \times 10 \wedge 9 / \mathrm{L}$ & $5.01(1.50)$ & $4.47(1.35)$ & $5.24(1.52)$ & 0.076 \\
\hline lymphocyte count, $\times 10 \wedge 9 / L$ & $1.60(0.55)$ & $1.30(0.35)$ & $1.72(0.58)$ & $0.008^{*}$ \\
\hline $\mathrm{CRP}, \mathrm{mg} / \mathrm{dL}$ & $9.69(13.77)$ & $22.65(18.19)$ & $4.18(5.66)$ & $<0.001^{*}$ \\
\hline $\mathrm{LDH}, \mathrm{U} / \mathrm{L}$ & $175.47(43.60)$ & $201.94(43.96)$ & $164.22(38.76)$ & $0.002^{*}$ \\
\hline CK, U/L & $91.95(118.16)$ & 133.18 (209.4) & $74.42(31.69)$ & 0.235 \\
\hline Lactic acid, mmol/L & $1.59(0.61)$ & $1.51(0.65)$ & $1.62(0.59)$ & 0.511 \\
\hline $\mathrm{PaO}_{2}$ to $\mathrm{FiO}_{2}$ ratio, $\mathrm{mmHg}$ & $308.67(94.40)$ & 198.47 (97.04) & $355.51(37.23)$ & $<0.001^{*}$ \\
\hline TSS on the worst chest CT scan & $4.28(4.26)$ & $8.59(4.15)$ & $2.45(2.73)$ & $<0.001^{*}$ \\
\hline TSS on chest CT on the 14th day after discharge & $1.75(2.23)$ & $3.94(2.33)$ & $0.83(1.39)$ & $<0.001^{*}$ \\
\hline glucocorticoids use & $16(28.1 \%)$ & $11(64.7 \%)$ & $5(12.5 \%)$ & $<0.001^{*}$ \\
\hline Total methyprednisolone dosage, mg & $213.75(323.87)$ & $289.09(370.4)$ & $48.0(17.89)$ & $0.019^{*}$ \\
\hline
\end{tabular}

Values are expressed as mean (SD)

*Statistically significant

time of testing during the study. No patient was reported having chronic respiratory diseases.

Among all subjects, seventeen were severe cases (29.8\%), forty were non-severe cases $(70.2 \%)$. There were mainly male patients(70.6\%)in the severe group, and the average age of patients was older compared with nonsevere cases. The mean Pao2/Fio2 ratio among severe cases was significantly lower than non-severe cases (198.47[SD, 97.04]; 355.51[SD, 37.23], P<0.001). Meanwhile, severe cases had higher serum lactate dehydrogenase (LDH), C-reactive protein (CRP) peaks and lower lymphocyte count compared with non-severe cases. But there was no significant difference in the values of white blood cells, creatine kinase (CK), lactic acid peaks and length of hospitalization between the two groups (Table 1).

\section{Lung function tests and respiratory muscle strength}

Table 2 presents the results of pulmonary function tests and respiratory muscle strength among COVID-19

Table 2 Results of pulmonary function tests and respiratory muscle strength among COVID-19 patients

\begin{tabular}{|c|c|c|c|c|}
\hline Parameter & Total $(n=57)$ & Severe $(n=17)$ & Non-severe $(n=40)$ & $p$ Value \\
\hline FVC (\% of predicted) & $100.96(15.93)$ & $95.92(19.59)$ & $103.10(13.83)$ & 0.12 \\
\hline FEV1 (\% of predicted) & $97.89(14.91)$ & $93.93(16.79)$ & $99.57(13.92)$ & 0.194 \\
\hline FEV1/FVC(\%) & $81.22(6.13)$ & $80.58(4.88)$ & $81.49(6.62)$ & 0.614 \\
\hline TLC (\% of predicted) & $93.94(12.75)$ & $88.72(16.20)$ & $96.22(10.35)$ & $0.048^{*}$ \\
\hline RV (\% of predicted) & $90.68(28.08)$ & $86.57(23.96)$ & $92.47(29.82)$ & 0.327 \\
\hline DLCO (\% of predicted) & 78.38 (13.59) & $74.14(18.85)$ & $80.12(10.56)$ & 0.139 \\
\hline Raw(\% of predicted) & $105.38(31.38)$ & $99.46(26.32)$ & $108.03(33.38)$ & 0.524 \\
\hline R5(\% of predicted) & $126.64(29.45)$ & 118.75 (29.98) & $130.00(28.96)$ & 0.072 \\
\hline R20(\% of predicted) & $132.76(30.95)$ & $120.15(31.46)$ & $138.12(29.50)$ & $0.024^{*}$ \\
\hline Pi max (\% of predicted) & $76.16(24.28)$ & $80.49(29.24)$ & $74.26(21.93)$ & 0.382 \\
\hline Pe max (\% of predicted) & $102.73(32.68)$ & $98.00(27.11)$ & $104.80(34.96)$ & 0.637 \\
\hline $6 \mathrm{MWD}, \mathrm{m}$ & $561.97(45.29)$ & $517.43(44.55)$ & $573.52(38.38)$ & $0.012^{*}$ \\
\hline 6MWD (\% predicted) & $94.61(6.55)$ & $88.46(7.61)$ & $96.20(5.31)$ & $0.011^{*}$ \\
\hline
\end{tabular}

Values are shown as mean (SD) severe vs non-severe with $p$ values *Statistically significant 
Table 3 The abnormal rate of pulmonary parameters and respiratory muscle strength between severe cases and mild cases

\begin{tabular}{|c|c|c|c|c|c|}
\hline Characteristic & Total $(n=57)$ & Severe $(n=17)$ & Non-severe $(n=40)$ & chisq & $p$ value \\
\hline FEV1 $<80 \%$ of pred & $5(8.8)$ & $3(17.6)$ & $2(5.0)$ & 1.066 & 0.302 \\
\hline FEV1 $\geq 80 \%$ of pred & $52(91.2)$ & $14(82.4)$ & $38(95.0)$ & & \\
\hline FVC $<80 \%$ of pred & $6(10.5)$ & $4(23.5)$ & $2(5.0)$ & 2.604 & 0.107 \\
\hline FVC $\geq 80 \%$ of pred & $51(89.5)$ & $13(76.5)$ & $38(95.0)$ & & \\
\hline FEV1 / FVC $<80 \%$ & $25(43.9)$ & $9(52.9)$ & $16(40.0)$ & 0.811 & 0.368 \\
\hline FEV1 / FVC $\geq 80 \%$ & $32(56.1)$ & $8(47.1)$ & $24(60.0)$ & & \\
\hline TLC $<80 \%$ of pred & $7(12.3)$ & $4(23.5)$ & $3(7.5)$ & 1.552 & 0.213 \\
\hline TLC $\geq 80 \%$ of pred & $50(87.7)$ & $13(76.5)$ & $37(92.5)$ & & \\
\hline DLCO $<80 \%$ of pred & $30(52.6)$ & $13(76.5)$ & $17(42.5)$ & 5.522 & $0.019^{*}$ \\
\hline DLCO $\geq 80 \%$ of pred & $27(43.4)$ & $4(23.5)$ & $23(57.5)$ & & \\
\hline $\mathrm{R} 5 \geq 150$ of pred & $8(14.0)$ & $2(11.8)$ & $6(15.0)$ & 0.103 & 0.554 \\
\hline R5 $<150$ of pred & $49(86.0)$ & $15(88.2)$ & $34(85.0)$ & & \\
\hline R20 $\geq 150$ of pred & $10(17.5)$ & $3(17.6)$ & $7(17.5)$ & 0.000 & 0.631 \\
\hline $\mathrm{R} 20<150$ of pred & $47(82.4)$ & $14(82.4)$ & $33(82.5)$ & & \\
\hline PImax $<80 \%$ of pred & $28(49.1)$ & $9(52.9)$ & $21(52.5)$ & 0.001 & 0.976 \\
\hline PImax $\geq 80 \%$ of pred & $29(50.9)$ & $8(47.1)$ & $19(47.5)$ & & \\
\hline PEmax $<80 \%$ of pred & $13(22.8)$ & $4(23.5)$ & $9(22.5)$ & 0.007 & 0.592 \\
\hline PEmax $\geq 80 \%$ of pred & $44(77.2)$ & $13(76.5)$ & $31(77.5)$ & & \\
\hline
\end{tabular}

pred predicted

*Statistically significant

patients. During follow-up at 1 month after hospital discharge, there were 30 individuals (52.6\%) with abnormal diffusion capacity among the 57 patients participating in our study. According to the ATS recommendations for evaluating respiratory impairment [8], twenty-six patients $(86.7 \%)$ had mild impairment of DLCO, while the other four (13.3\%) had moderate impairment. There was significant difference in impaired diffusing-capacity between the two groups, which accounted for $42.5 \%$ in non-severe cases, and $75.6 \%$ in severe cases, respectively $(p<0.05$, Table 3$)$.

The group means of forced expiratory volume in $1 \mathrm{~s}$ (FEV1), static lung volumes were within normal limits ( $>80 \%$ predicted). However, several cases of abnormalities in FVC, FEV1, and FEV1/FVC ratio were detected. Five patients $(8.7 \%)$ had mild impairment of FVC, one (1.8\%) had moderate impairment of FVC, 5 (8.7\%) had mild impairment of FEV1, and 25 (43.9\%) had mild impairment of FEV1/FVC. There were 8 patients (14.0\%) and 10 patients (17.5\%) had increased R5 and R20 more than $150 \%$ of the predicted value, respectively. Up to $12.2 \%(n=7)$ of patients had reduction in parameters of lung volume (TLC) at 1 month. Among them, 6 had mild impairment, one had moderate impairment. TLC declined more significantly in severe cases $(p=0.048)$. There was no difference in FVC, FEV1, and FEV1/FVC between the two groups. Table 4 shows the detailed pulmonary function data of all 57 subjects. The majority of the impairment in FEV1 and FVC suggests a restrictive abnormality. One patient without history of asthma had obstructive abnormality with a FEV1/FVC ratio $<70 \%$ predicted (up to $72 \%$ after bronchodilation), who had significant history of cigarette smoking. Although no complained of symptoms of asthma, one other patient had a significant bronchodilator response with increments of FEV1 $>200 \mathrm{ml}$ after inhalation of salbutamol.

More than half of the subjects had impairment in respiratory muscle strength. There were 28 patients (49.1\%) and 13 patients (22.8\%) had Pimax and Pemax values less than $80 \%$ of the predicted value, respectively.13 patients had moderate impairment of respiratory muscle strength, of whom 11 were non-severe cases (Table 4). When grouped by the administration of steroid, no statistical significance was found in respiratory muscle strength between the glucocorticoid group and the regular group (Table 5).

\section{Chest radiographs and correlations with lung function}

During follow-up at 30 days after discharge, six patients (10.5\%) complained of slight cough, four $(7.0 \%)$ had shortness of breath, and three (5.3\%) had occasional wheezing. Follow-up CT scan at this time showed that 31 patients $(54.4 \%)$ had residual abnormality, of which 16 were severe cases $(94.1 \%)$ and 15 were non-severe cases (37.5\%).Most of the residual imaging abnormalities was patchy ground glass opacity with periphery 
Table 4 Clinical and Pulmonary Function Data of COVID-19 Patients $(n=57)$

\begin{tabular}{|c|c|c|c|c|c|c|c|c|c|}
\hline Patient No & Age, yr & Clinical type & FVC & FEV 1 & FEV $1 \%$ FVC & DLCO SB & TLC & R 5 & R20 \\
\hline 1 & 41 & 2 & 85.80 & 90.40 & 90.57 & 77.90 & 92.60 & 99.10 & 103.40 \\
\hline 2 & 36 & 2 & 114.20 & 114.50 & 86.71 & 79.40 & 110.90 & 113.60 & 153.50 \\
\hline 3 & 29 & 2 & 103.80 & 105.00 & 87.81 & 72.20 & 100.00 & 112.50 & 102.30 \\
\hline 4 & 65 & 1 & 90.60 & 88.50 & 77.09 & 71.50 & 81.00 & 122.70 & 117.60 \\
\hline 5 & 52 & 1 & 75.90 & 75.90 & 80.77 & 82.00 & 69.90 & 197.70 & 195.70 \\
\hline 6 & 36 & 1 & 75.70 & 80.40 & 89.21 & 68.00 & 81.80 & 117.10 & 117.20 \\
\hline 7 & 56 & 2 & 72.00 & 74.60 & 87.38 & 64.20 & 70.70 & 144.20 & 167.10 \\
\hline 8 & 33 & 2 & 114.50 & 114.10 & 83.82 & 71.70 & 98.40 & 91.20 & 112.50 \\
\hline 9 & 54 & 1 & 94.30 & 89.30 & 79.95 & 60.60 & 83.50 & 108.60 & 101.30 \\
\hline 10 & 42 & 1 & 84.60 & 83.60 & 81.67 & 92.50 & 78.70 & 172.50 & 171.00 \\
\hline 11 & 38 & 2 & 102.70 & 94.90 & 79.88 & 77.20 & 93.70 & 97.50 & 99.60 \\
\hline 12 & 33 & 2 & 86.80 & 90.00 & 89.78 & 67.50 & 94.60 & 125.10 & 137.60 \\
\hline 13 & 69 & 1 & 88.00 & 86.00 & 75.27 & 48.60 & 77.40 & 113.00 & 112.70 \\
\hline 14 & 37 & 2 & 74.40 & 62.00 & 71.88 & 71.80 & 77.30 & 146.50 & 167.40 \\
\hline 15 & 20 & 2 & 85.70 & 73.70 & 79.04 & 78.60 & 82.00 & 121.60 & 159.60 \\
\hline 16 & 71 & 1 & 91.90 & 88.00 & 79.50 & 49.20 & 89.30 & 109.60 & 90.60 \\
\hline 17 & 36 & 1 & 83.00 & 83.20 & 82.98 & 72.80 & 79.40 & 76.10 & 85.90 \\
\hline 18 & 33 & 2 & 95.00 & 98.10 & 89.22 & 72.80 & 100.30 & 225.20 & 202.70 \\
\hline 19 & 37 & 2 & 96.60 & 108.00 & 96.25 & 70.00 & 108.20 & 178.30 & 189.60 \\
\hline 20 & 63 & 2 & 93.30 & 84.90 & 76.31 & 72.40 & 91.00 & 115.30 & 93.90 \\
\hline 21 & 37 & 2 & 118.20 & 93.70 & 68.55 & 80.10 & 103.80 & 134.20 & 107.80 \\
\hline 22 & 60 & 1 & 59.70 & 64.30 & 84.89 & 50.10 & 53.20 & 111.50 & 117.90 \\
\hline 23 & 56 & 2 & 103.40 & 102.00 & 79.22 & 70.90 & 90.60 & 114.40 & 138.70 \\
\hline 24 & 32 & 1 & 86.80 & 85.30 & 85.33 & 53.30 & 106.40 & 86.90 & 86.50 \\
\hline 25 & 36 & 2 & 101.00 & 100.40 & 85.89 & 64.00 & 91.70 & 161.50 & 148.70 \\
\hline 26 & 62 & 1 & 100.90 & 92.90 & 77.07 & 72.90 & 97.70 & 168.50 & 128.40 \\
\hline 27 & 39 & 2 & 106.90 & 93.30 & 72.55 & 66.80 & 92.90 & 78.20 & 86.40 \\
\hline 28 & 29 & 2 & 113.30 & 100.50 & 74.41 & 74.70 & 108.10 & 110.10 & 135.70 \\
\hline 29 & 22 & 2 & 75.60 & 85.70 & 98.91 & 65.40 & 122.90 & 107.60 & 103.80 \\
\hline 30 & 25 & 2 & 98.60 & 99.20 & 85.31 & 79.00 & 92.40 & 139.60 & 158.70 \\
\hline 31 & 64 & 2 & 111.60 & 106.40 & 79.28 & 73.80 & 100.40 & 127.00 & 131.80 \\
\hline 32 & 32 & 2 & 116.30 & 106.00 & 78.92 & 68.60 & 106.60 & 240.10 & 183.70 \\
\hline 33 & 56 & 2 & 111.60 & 105.30 & 80.22 & 79.10 & 98.80 & 147.20 & 134.90 \\
\hline 34 & 62 & 1 & 134.90 & 128.60 & 75.45 & 119.60 & 117.00 & 114.30 & 88.60 \\
\hline 35 & 39 & 2 & 109.10 & 92.10 & 73.04 & 89.20 & 106.20 & 126.90 & 125.50 \\
\hline 36 & 53 & 1 & 104.00 & 99.90 & 78.96 & 87.00 & 96.30 & 142.70 & 70.20 \\
\hline 37 & 29 & 2 & 96.90 & 84.10 & 75.20 & 92.50 & 96.70 & 126.50 & 100.90 \\
\hline 38 & 22 & 1 & 111.70 & 111.40 & 77.85 & 81.50 & 91.70 & 150.50 & 73.10 \\
\hline 39 & 25 & 2 & 106.50 & 98.20 & 78.22 & 84.40 & 91.90 & 87.90 & 80.10 \\
\hline 40 & 66 & 2 & 89.20 & 88.60 & 77.03 & 96.10 & 80.30 & 119.80 & 98.30 \\
\hline 41 & 56 & 2 & 133.60 & 125.60 & 78.00 & 98.10 & 103.40 & 131.30 & 111.60 \\
\hline 42 & 64 & 1 & 127.90 & 117.30 & 75.16 & 81.20 & 114.80 & 139.30 & 110.40 \\
\hline 43 & 56 & 1 & 121.20 & 112.10 & 76.98 & 81.30 & 102.50 & 127.10 & 103.30 \\
\hline 44 & 36 & 2 & 106.90 & 114.20 & 88.53 & 80.00 & 91.80 & 121.90 & 120.70 \\
\hline
\end{tabular}


Table 4 Clinical and Pulmonary Function Data of COVID-19 Patients $(n=57)$ (Continued)

\begin{tabular}{llllllllll}
\hline Patient No & Age, yr & Clinical type & FVC & FEV 1 & FEV 1 \% FVC & DLCO SB & TLC & R 5 & R20 \\
\hline 45 & 49 & 2 & 111.10 & 108.30 & 82.66 & 82.90 & 108.80 & 129.50 & 144.80 \\
46 & 38 & 1 & 99.50 & 110.10 & 91.75 & 82.10 & 87.50 & 94.90 & 115.30 \\
47 & 39 & 2 & 90.80 & 87.20 & 79.87 & 107.50 & 81.20 & 151.30 & 159.50 \\
48 & 44 & 2 & 106.60 & 105.20 & 80.86 & 96.50 & 96.80 & 142.90 & 153.00 \\
49 & 29 & 2 & 96.00 & 91.20 & 79.98 & 92.30 & 91.60 & 118.70 & 139.70 \\
50 & 59 & 2 & 109.50 & 103.60 & 79.77 & 92.70 & 93.80 & 127.20 & 152.50 \\
51 & 55 & 2 & 114.00 & 124.00 & 86.51 & 91.50 & 96.00 & 117.20 & 121.00 \\
52 & 63 & 2 & 109.50 & 104.10 & 79.15 & 80.30 & 92.00 & 128.70 & 135.70 \\
53 & 57 & 2 & 116.80 & 114.60 & 82.91 & 89.80 & 99.10 & 90.60 & 115.00 \\
54 & 59 & 2 & 96.20 & 91.30 & 79.66 & 86.00 & 96.30 & 137.20 & 143.70 \\
55 & 55 & 2 & 129.30 & 124.60 & 81.32 & 82.80 & 113.80 & 116.40 & 142.10 \\
56 & 65 & 2 & 101.20 & 107.90 & 83.10 & 84.80 & 86.00 & 103.80 & 99.50 \\
57 & 63 & 2 & 119.50 & 115.20 & 81.19 & 85.30 & 95.10 & 137.50 & 145.70 \\
\hline
\end{tabular}

Values given as \% predicted, unless otherwise indicated. Clinical type, 1 for severe, 2 for non-severe

distribution, which had obvious absorption compared with the worst chest CT scan (Fig. 2.a-b). Four patients had pulmonary fibrosis (Fig. 2.c-d), all of whom were severe patients. Compared with non-severe cases, severe patients had a significantly higher CT score (3.94[SD, 2.23]; 0.83 [SD, 1.39]; $p<0.01$ ). At the acute phase, lung total severity score was negatively correlated with TLC and R20 ( $P=0.049,0.044$, Fig. 3), but the correlation disappeared during follow-up period.

\section{6-min walk test}

The mean 6-min walking distance (6MWD) in all subjects was $561.97 \mathrm{~m}( \pm 45.29 \mathrm{~m})$. Severe patients had a shorter $6 \mathrm{~min}$ walking distance than non-severe patients $(517.43 \mathrm{~m}$ [SD, $44.55 \mathrm{~m}$ ]; $573.52 \mathrm{~m}$ [SD, $38.38 \mathrm{~m}$ ], $P=$ 0.012 ). And the 6MWD of severe cases reached only $88.4 \%$ of the predicted values, which was significant lower than non-severe cases $(p=0.011$, Table 2$)$.

\section{Discussion}

Since COVID-19 broke out worldwide over the last 6 months, the mechanism, clinical characteristics, prognosis and effective treatment of the disease had not yet been adequately elucidated despite the great efforts that had been extended. Recent research and our date showed that nearly half of the discharged patients had residual abnormality in chest CT scan [6]. Global concerns are raised regarding the assessment of the lung injury for discharged patients. This study showed that in early convalescence, approximately three-quarters of patients with COVID-19 developed pulmonary function impairment, the most common of which was impaired diffusing-capacity and the decline in FEV1/FVC ratio.

DLCO abnormalities occurred in more than half of the COVID-19 patients, the data indicated impaired diffusion pathways in the intra-alveolar. To date, no other follow-up data on lung function in patients with COVID-19 can be compared. S.A. MEO et al. reported that severe acute respiratory syndrome (SARS) and COVID-19 had similar biological and clinical characteristics [9]. Previous studies on SARS survivors showed that impaired DLCO was the most common abnormality, ranging from 15.5 to $43.6 \%$ [10-15]. Our results were consistent with them. Autopsy on patients died from COVID-19 showed different degrees of destruction in alveolar structure, and pulmonary interstitial fibrosis were observed [16, 17].. Pathological changes in lungs can explain the impaired DLCO to a certain extent. Compared with non-severe case, severe patients were more likely to have DLCO abnormalities. Surprisingly, a small percentage of patients with no residual imaging abnormalities also experienced a slight decrease in DLCO. We think that these patients might have abnormal tiny blood vessels or microthrombus formation. Long-term follow-up studies of SARS survivors had shown that DLCO might remain abnormal within 3 years of recovery in some patients [18]. We will continue to perform long-term follow-up on these patients to see the trend of DLCO impairment.

Our results showed that six patients (10.5\%) had obstructive pulmonary dysfunction and $7(12.3 \%)$ had restrictive ventilation dysfunction. Two severe subjects had residual combined restrictive and obstructive type of functional impairment. Series articles on SARS survivors reporting very low rates of either obstruction or restriction, which was consistent with our research data $[10,11]$. Pathological findings showed that mucous plugs were found in small airway in some severe COVID-19 patients [17], which could explain the declined ventilatory function 
Table 5 Results of pulmonary function tests and respiratory muscle strength among COVID-19 patients between glucocorticoid group and the regular groups

\begin{tabular}{|c|c|c|c|c|}
\hline Parameter & All patients $(n=57)$ & GC group $(n=16)$ & Regular group $(n=41)$ & $p$ Value \\
\hline FVC (\% of predicted) & $100.96(15.93)$ & $97.25(18.69)$ & $102.40(14.72)$ & 0.414 \\
\hline FEV1 (\% of predicted) & $97.89(14.91)$ & $94.35(15.40)$ & $99.27(14.68)$ & 0.279 \\
\hline FEV1 / FVC (\%) & $81.22(6.13)$ & $80.74(4.68)$ & $81.40(6.65)$ & 0.804 \\
\hline TLC (\% of predicted) & $93.94(12.75)$ & $90.15(16.01)$ & $95.45(11.06)$ & 0.323 \\
\hline RV (\% of predicted) & $90.68(28.08)$ & $85.49(19.01)$ & $92.76(30.95)$ & 0.593 \\
\hline DLCO (\% of predicted & $78.38(13.59)$ & $74.67(14.37)$ & $79.78(13.20)$ & 0.657 \\
\hline Raw (\% of predicted) & $105.38(31.38)$ & $96.02(25.81)$ & $109.22(32.93)$ & 0.214 \\
\hline R5 (\% of predicted) & $126.64(29.45)$ & $119.66(30.62)$ & $129.37(28.91)$ & 0.127 \\
\hline R20 (\% of predicted) & $132.76(30.95)$ & $123.51(31.99)$ & $136.37(30.15)$ & 0.106 \\
\hline Pimax (\% of predicted) & $76.16(24.28)$ & $85.21(26.54)$ & $72.53(22.65)$ & 0.059 \\
\hline Pemax (\% of predicted) & $102.73(32.68)$ & $104.22(28.03)$ & $102.14(34.68)$ & 0.479 \\
\hline
\end{tabular}

Values given as \% predicted, unless otherwise indicated

GC glucocorticoid

to an extent. In addition to acute lung injury, neuromuscular weakness could also lead to decreased lung function. Certainly, a few patients suffered from lower FEV1 or FEV1/FVC ratio might due to long-term smoking or untypical airway hyperresponsiveness.

Surprisingly, during the early rehabilitation phase, lung total severity score had no significant correlation with FEV1, FVC or DLCO (Fig. 3), which was inconsistent with researches on SARS survivors [19]. It seems that the impairment of lung function was not necessarily agreed with the severity of illness or residual imaging changes. It was an interesting finding. We speculate that it was because most severe patients used glucocorticoid during hospitalization, suggesting that corticosteroids may improve the prognosis of patients with COVID-19. But most of the subjects in our hospital were imported cases from Hubei, small sample size and selection bias might affect statistical outcome. Besides, our CT
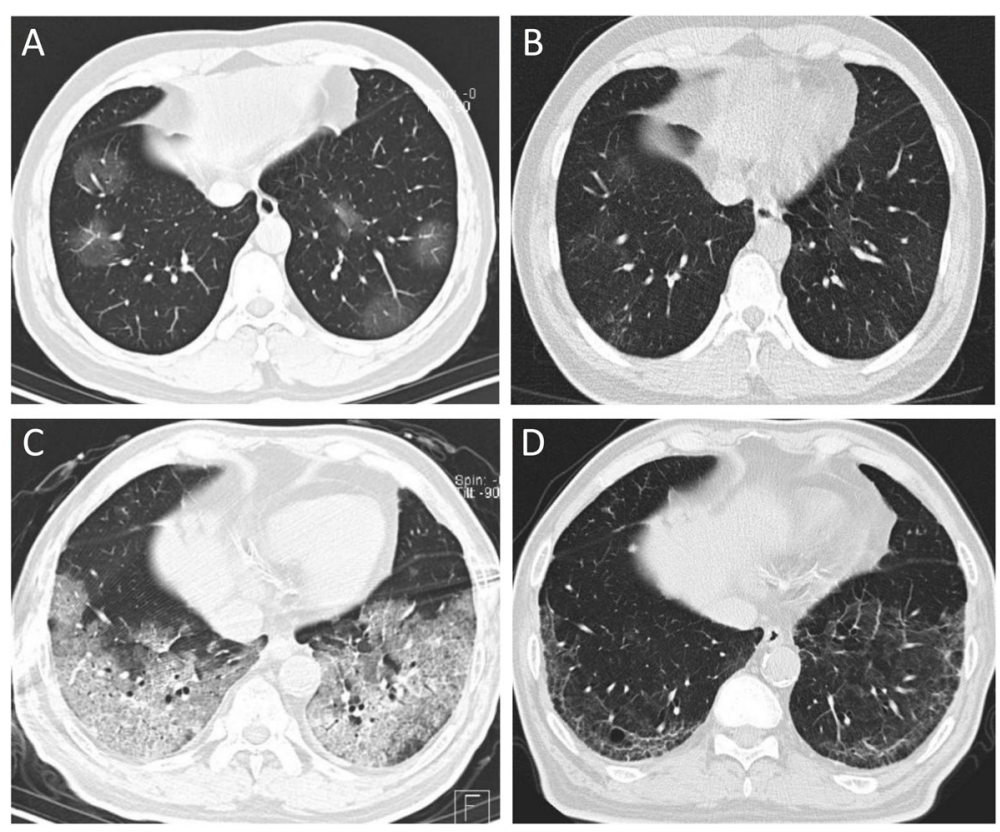

Fig. 2 HRCT scan of a 44-year-old man in acute stage demonstrated bilateral peripheral ground-glass opacities (GGO). Lung total severity score (TSS)was 7. B. Follow-up CT of the same patient at 30 days after discharge from hospital showed that patchy ground glass opacity had obvious absorption.TSS was 3 .C. Worst CT scan of a severe patient during acute stage showed diffuse GGO, consolidation also could be seen in some area. TSS was 13. D: HRCT scan of the same patient obtained 30 days after discharge showed peripheral fibrosis consists of irregular linear opacities. Concomitant presence of GGO was also visible.TSS is 5 


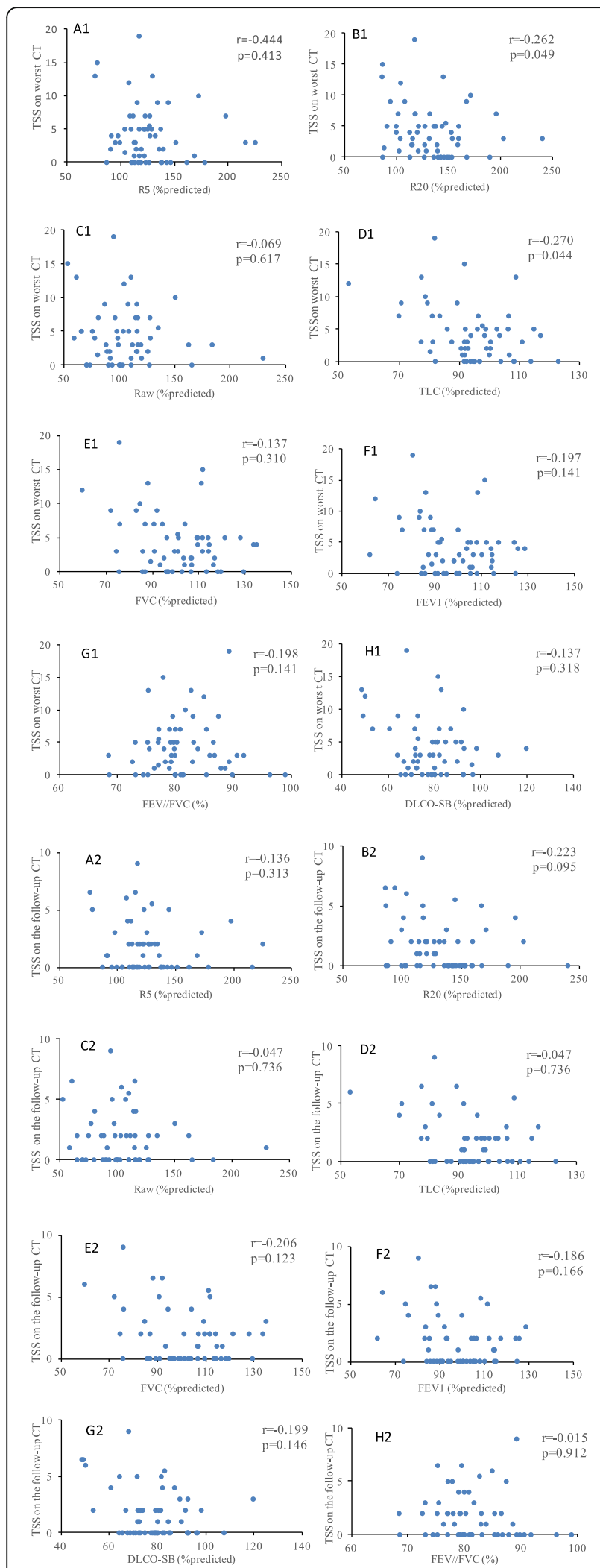

Fig. 3 Correlation of total severity score on worst $C T$ (from $\mathbf{a} 1$ to $\mathbf{h} 1$ ) and follow-up CT (from a2 to h2) with pulmonary function parameters. quantitative evaluation was not actual represented the percentage of lung parenchyma that showed evidence of abnormalities. According to the criteria, in the same lung lobe, lung inflammatory lesions area within $25 \%$ of the difference was possible to be calculated as the same score, which might influence statistical results. In the next step, we will perform long-term follow-up study and expand the sample size to see whether similar correlation conclusions still exist.

More than half of the patients experienced a decrease in respiratory muscle strength. Approximately $29.8 \%$ of patients in our study were severe or critical, who had hypoxemia during hospitalization, requiring supplemental oxygen and bed rest, and prolonged bed rest might lead to muscle disorders. In addition, systemic use of corticosteroids might cause steroid myopathy. But when grouped by the administration of steroid, no statistical significance was found in respiratory muscle strength between the glucocorticoid group and the regular group. This result indicated that corticosteroid was not the main cause of respiratory muscle weakness. In fact, there was no difference on declining respiratory muscle strength between severe and non-severe groups. However, the direct effect of virus on respiratory muscles needs further research.

In early convalescence, the 6MWD of the severe patients was significantly shorter than that of the nonsevere patients, indicating that the severe patients have poor exercise tolerance. Besides the impaired TLC and worse DLCO in severe group, we should also pay attention to cardiac function of the patients. Exercise cardiopulmonary function should be performed in further studies. Series articles on SARS survivors showed the impaired lung function existed till 1 year [10, 11]. Longer follow-up on COVID-19 patients should be made to observe the characteristic and change tendency of lung function and exercise tolerance.

There are several limitations to this study. Firstly, this is a cross-sectional study with small sample size in stratified analysis, only provides a short follow-up. The heterogeneity of our findings is not comprehensive. Secondly, only 57 of 102 COVID-19 patients (56\%) in our hospital had completed the serial assessments, and the results might not be representative of the entire group. Lastly, although full lung function tests and $6 \mathrm{MWT}$ were conducted in our patients, we did not perform cardiopulmonary exercise testing, as many patients complained of generalized muscle weakness on followup. Meanwhile, labor intensity of CPET might be too high for patients in early recovery period.

In conclusion, impaired diffusing-capacity, respiratory muscle strength decrease, and lung imaging abnormalities were detected in more than half of the COVID-19 patients in early convalescence phase. Compared with non-severe cases, severe patients had a higher incidence 
of DLCO impairment and encountered more TLC decrease and 6MWD decline. Longer follow-up studies in COVID-19 patients should be performed to investigate the clinical outcome of recovered COVID-19 patients.

\section{Abbreviation}

BMI: Body mass index; CK: Creatine kinase; CRP: C-reactive protein; WBC: White blood cell count; LDH: Lactate dehydrogenase; COVID19: Coronavirus disease 2019; DLCO: Diffusing capacity of the lung for carbon monoxide; FVC: Forced vital capacity; FEV1: Forced expiratory volume in $1 \mathrm{~s}$; LOS: Length of hospital stay; PImax: Maximum static inspiratory pressures; PEmax: Maximum static expiratory pressures; Raw: Airway resistant; R5: Airway resistance at an oscillation frequency of $5 \mathrm{~Hz}$; R20: Airway resistance at an oscillation frequency of $20 \mathrm{~Hz}$; TLC: Total lung capacity; TSS: Total severity score; 6MWD: 6 min walk distance; 6MWT: 6-min walk test

\section{Acknowledgements}

Yiying Huang, Cuiyan Tan and Jian Wu contributed equally to this work.

\section{Authors' contributions}

$\sqcup$ designed the study, WJ performed the research, TCY collected the data, HYY analysed data and wrote the paper. All authors read and approved the final manuscript.

\section{Availability of data and materials}

All data generated or analysed during this study are included in this published article.

\section{Ethics approval and consent to participate}

This study was approved by the institutional ethics committee of the Fifth Affiliated Hospital of Sun Yat-sen University.

\section{Consent for publication}

Consent for publication is not applicable.

\section{Competing interests}

The authors declare that they have no competing interests.

\section{Author details}

${ }^{1}$ Department of Pulmonary and Critical Care Medicine, The Fifth Affiliated Hospital of Sun Yat-sen University, 52 East Meihua Rd, Zhuhai City 519000, China. ${ }^{2}$ Guangdong Provincial Key Laboratory of Biomedical Imaging, The Fifth Affiliated Hospital of Sun Yat-sen University, 52 East Meihua Rd, Zhuhai City 519000, China. ${ }^{3}$ Department of Cardiovascular Medicine, The Fifth Affiliated Hospital of Sun Yat-sen University, Zhuhai, China.

Received: 30 April 2020 Accepted: 18 June 2020

Published online: 29 June 2020

\section{References}

1. Chen N, Zhou M, Dong X, Qu J, Gong F, Han Y, Qiu Y, Wang J, Liu Y, Wei Y, et al. Epidemiological and clinical characteristics of 99 cases of 2019 novel coronavirus pneumonia in Wuhan, China: a descriptive study. Lancet. 2020; 395(1)507-13.

2. Huang C, Wang Y, Li X, Ren L, Zhao J, Hu Y, Zhang L, Fan G, Xu J, Gu X, et al. Clinical features of patients infected with 2019 novel coronavirus in Wuhan, China. Lancet. 2020:395(10223):497-506.

3. Ding Q, Lu P, Fan Y, Xia Y, Liu M. The clinical characteristics of pneumonia patients coinfected with 2019 novel coronavirus and influenza virus in Wuhan, China. J Med Virol. 2020.

4. Chung M, Bernheim A, Mei X, Zhang N, Huang M, Zeng X, Cui J, Xu W, Yang Y, Fayad ZA, et al. CT Imaging Features of 2019 Novel Coronavirus (2019-nCoV). Radiology. 2020;295(1):202-7.

5. James H, MT. Hypothesis: angiotensin-converting enzyme inhibitors and angiotensin receptor blockers may increase the risk of severe COVID-19. J Travel Med. 2020:1-2.

6. Li K, Fang Y, Li W, Pan C, Qin P, Zhong Y, Liu X, Huang M, Liao Y, Li S. CT image visual quantitative evaluation and clinical classification of coronavirus disease (COVID-19). Eur Radiol. 2020.
7. SHERRILL PLEA. Reference equations for the six-minute walk in healthy adults. Am J Respir Crit Care Med. 1998;158:1384-7.

8. Wang CJ, Ng CY, Brook RH. Response to COVID-19 in Taiwan: big data analytics, new technology, and proactive testing. JAMA. 2020;323(14):133940.

9. Meo SA, Alhowikan AM, Al-Khlaiwi T, Meo IM, Halepoto DM, labal M, Usmani AM, Hajjar W, Ahmed N. Novel coronavirus 2019-nCoV: prevalence, biological and clinical characteristics comparison with SARS-CoV and MERSCoV. Eur Rev Med Pharmaco. 2020;24(4):2012.

10. Hui DS, Wong KT, Ko FW, Tam LS, Chan DP, Woo J, Sung JJY. The 1-year impact of severe acute respiratory syndrome on pulmonary function, exercise capacity, and quality of life in a cohort of survivors. Chest. 2005; 128(4):2247-61.

11. Ong K, Ng AW, Lee LS, Kaw G, Kwek S, Leow MK, Earnest A. 1-year pulmonary function and health status in survivors of severe acute respiratory syndrome. Chest. 2005;128(3):1393-400.

12. AWNL K-CO. Pulmonary function and exercise capacity in survivors of severe acute respiratory syndrome. Eur Respir J. 2004;24:436-42 2004.

13. Su MC, Hsieh YT, Wang YH, Lin AS, Chung YH, Lin MC. Exercise capacity and pulmonary function in hospital workers recovered from severe acute respiratory syndrome. Respiration. 2007;74(5):511-6.

14. Xie L, Liu Y, Fan B, Xiao Y, Tian Q, Chen L, Zhao H, Chen W. Dynamic changes of serum SARS-coronavirus IgG, pulmonary function and radiography in patients recovering from SARS after hospital discharge. Resp Res. 2005;6(1):5.

15. Zheng Z, Chen R, Wu H, Liu X, He W, Xu Y, Chen S, Li Y, Zheng J, Zhong N. Changes in pulmonary function in severe acute respiratory syndrome patients during convalescent period. Zhongguo Wei Zhong Bing Ji Jiu Yi Xue. 2005;17(6):329.

16. Yao X, Li T, He Z, Ping Y, Liu H, Yu S, Mou H, Wang L, Zhang H, F u W, et al. A pathological report of three COVID-19 cases by minimally invasive autopsies. medRxiv, https://doi.org/10.3760/cma.j.cn112151-20200312-00193,

17. Xu Z, Shi L, Wang Y, Zhang J, Huang L, Zhang C, Liu S, Zhao P, Liu H, Zhu L, et al. Pathological findings of COVID-19 associated with acute respiratory distress syndrome. Lancet Respir Med. 2020:8(4):420-2.

18. NGAI JC, Ko FW, Ng SS, To K-W, Tong M, Hui DS. The long-term impact of severe acute respiratory syndrome on pulmonary function, exercise capacity and health status. Respirology. 2010;15(3):543-50.

19. Hsian-He Hsu M, Ching Tzao MP, Chin-Pyng Wu MP, Wei-Chou Chang M, Chen-Liang Tsai M, Ho-Jui Tung P, Cheng-Yu Chen M. Correlation of highresolution $C T$, symptoms, and pulmonary function inpatients during recovery from severe acute respiratory syndrome, symptoms, and pulmonary function in. Chest. 2004;126:149-58.

\section{Publisher's Note}

Springer Nature remains neutral with regard to jurisdictional claims in published maps and institutional affiliations.

Ready to submit your research? Choose BMC and benefit from

- fast, convenient online submission

- thorough peer review by experienced researchers in your field

- rapid publication on acceptance

- support for research data, including large and complex data types

- gold Open Access which fosters wider collaboration and increased citations

- maximum visibility for your research: over $100 \mathrm{M}$ website views per year

At $\mathrm{BMC}$, research is always in progress.

Learn more biomedcentral.com/submissions 